\title{
Photoluminescence and far-infrared absorption in Si-doped self-organized InAs quantum dots
}

\author{
J. Phillips, K. Kamath, X. Zhou, N. Chervela, and P. Bhattacharya ${ }^{\text {a) }}$ \\ Solid State Electronics Laboratory, Department of Electrical Engineering and Computer Science, \\ The University of Michigan, Ann Arbor, Michigan 48109-2122
}

(Received 23 June 1997; accepted for publication 6 August 1997)

\begin{abstract}
We report far-infrared absorption in directly doped self-organized InAs quantum dots. Photoluminescence spectra demonstrate a blue shift in peak intensity for increasing doping in the quantum dots. Far-infrared absorption measurements using a Fourier transform infrared spectrometer show absorption in the range of $13-18 \mu \mathrm{m}$ for quantum dots with $\mathrm{Al}_{0.15} \mathrm{Ga}_{0.85} \mathrm{As}$ and GaAs as the barrier material. (C) 1997 American Institute of Physics. [S0003-6951(97)02541-2]
\end{abstract}

There is currently a need for sources and detectors in the mid and far-infrared spectral regions for applications such as IR spectroscopy for chemical analysis, remote sensing, and atmospheric communications. Semiconductor devices based on band-to-band transitions, in $\mathrm{HgCdTe}$ and similar materials, have had some success in this regime, but are often dominated by Auger recombination and need to be cooled below room temperature. For this reason, many have looked to semiconductor devices based on intersubband transitions in quantum confined structures. Quantum well devices such as intersubband detectors ${ }^{1-4}$ and the quantum cascade laser ${ }^{5,6}$ have demonstrated much success in this regime. Quantum dots provide two unique advantages for this application: the intersubband relaxation times (which control detector noise) in this nanostructures are $\sim 100 \mathrm{ps}$ at room temperature due to reduced electron-photon interaction ${ }^{7}$ and, unlike quantum wells, quantum dots are sensitive to normal incidence photoexcitation. With recent success in fabricating high quality quantum dots through self-organized growth, ${ }^{8-11}$ it is of immense interest to examine the intersubband absorption characteristics of these structures. Far-infrared absorption has been reported for charged InGaAs/GaAs quantum dots ${ }^{12}$ for wavelengths $>20 \mu \mathrm{m}$. Midinfrared photoconductivity has been reported for delta-doped InAs/AlGaAs quantum dots near $3 \mu \mathrm{m}$ for subband to continuum transitions. ${ }^{13}$ We believe this is the first report of far-infrared absorption in quantum dots in the range of 10-20 $\mu \mathrm{m}$, important for spectroscopy and image recognition.

Directly doped InAs quantum dots grown on GaAs and $\mathrm{Al}_{0.15} \mathrm{Ga}_{0.85} \mathrm{As}$ were studied. The samples were grown on semi-insulating $\mathrm{GaAs}(100)$ substrates in a Varian Gen II molecular beam epitaxy (MBE) system. The GaAs or $\mathrm{Al}_{0.15} \mathrm{Ga}_{0.85} \mathrm{As}$ barrier layers were first grown, following a GaAs buffer layer, at a substrate temperature of $620^{\circ} \mathrm{C}$. The substrate temperature was then ramped to $500{ }^{\circ} \mathrm{C}$ during the end of the barrier layer growth with a $10 \mathrm{~s}$ pause prior to the InAs growth. A nominal thickness of $8.4 \AA$ of InAs was then grown at a rate of $0.3 \AA / s$. Examination of the reflection high-energy electron diffraction (RHEED) spectrum during growth showed a transition from streaked to spotty, indicating 3D island formation after about $18 \mathrm{~s}$ of growth in all cases corresponding to a nominal thickness of $5.6 \AA$ Af InAs.

${ }^{a)} \mathrm{pkb} @$ eecs.umich.edu
Silicon was continually supplied during the InAs growth for doping levels ranging from nominally undoped to $n=4$ $\times 10^{18} \mathrm{~cm}^{-3}$. A $5 \mathrm{~s}$ pause was then given to further allow island formation following InAs growth. GaAs or $\mathrm{Al}_{0.15} \mathrm{Ga}_{0.85} \mathrm{As}$ barrier layers were then grown at a substrate temperature of $620^{\circ} \mathrm{C}$ which was ramped at the beginning of the barrier layer growth. The schematic of a typical heterostructure is shown in Fig. 1. Similar samples with quantum dots grown on the surface were examined with atomic force microscopy (AFM) indicating an estimated base dimension of $150 \AA$, height of $70 \AA$, and a dot density $10^{11} \mathrm{~cm}^{-2}$. The doped samples were grown consecutively to avoid growth fluctuations in order to obtain reproducible dot formation.

The samples were first characterized by photoluminescence (PL) measurements at a temperature of $18 \mathrm{~K}$ with a $\mathrm{HeNe}(\lambda=632.8 \mathrm{~nm})$ laser, lock-in amplification, a $1 \mathrm{~m}$ spectrometer, and a cooled photomultiplier tube for detection. The PL spectra for InAs/GaAs quantum dots (QDs) with various doping levels under the same excitation power density demonstrate a blue shift in peak intensity with increasing doping (Fig. 2), due likely to state filling effects in the quantum dot. This is contrary to a recent report which shows a red shift in PL with increasing doping for modulation doped InAs/GaAs quantum dots, which was attributed to band gap renormalization. ${ }^{14}$ For increasing doping, an increase in linewidth and decrease in peak intensity is observed, most likely due to the incorporation of nonradiative impurities. For samples grown on $\mathrm{Al}_{0.15} \mathrm{Ga}_{0.85} \mathrm{As}$, the PL peak intensity is observed at shorter wavelengths as compared to GaAs (Fig. 3) due to the increased barrier height. In all cases, only a single peak is observed in the PL spectra of the dots. With

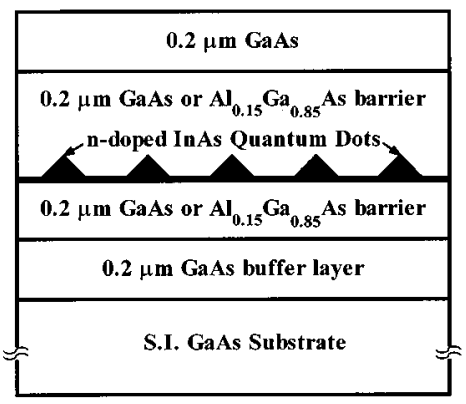

FIG. 1. Schematic of MBE growth structure for photoluminescence and far-infrared absorption spectra measurements. 


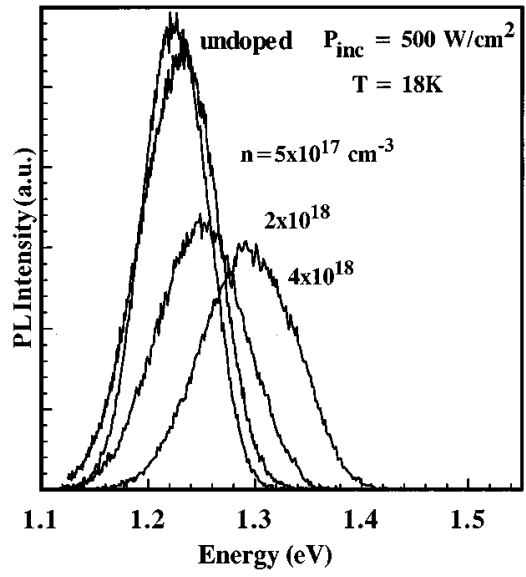

FIG. 2. Photoluminescence spectra of InAs/GaAs quantum dots of varying doping levels.

increasing excitation, a blue shift in PL peak intensity is observed, but clearly defined peaks corresponding to excited state transitions in the quantum dots are not observed.

For far-infrared absorption measurements, a multireflection waveguide geometry was used to increase net absorption, ${ }^{15}$ as shown schematically in Fig. 4(a). Waveguides of dimensions $1.2 \mathrm{~cm} \times 1 \mathrm{~cm}$ were fabricated with polished backsides and polished $45^{\circ}$ facets for optimum light input and output. Absorption measurements were made with a MATTSON Cygnus 100 Fourier transform infrared (FTIR) system with a lamp source and a $\mathrm{LN}_{2}$ cooled wideband $\mathrm{HgCdTe}$ detector. The resolution of the measurements taken is $2 \mathrm{~cm}^{-1}$ corresponding to $0.25 \mathrm{meV}$. Absorbance was determined by

$$
A=\log \left(\frac{S_{\mathrm{GaAs}}}{S_{\text {sample }}}\right),
$$

where $S_{\text {sample }}$ and $S_{\mathrm{GaAs}}$ are the single beam outputs of the sample to be measured and a waveguide fabricated from the same GaAs substrate as the grown structure, respectively. Due to the spectral nature of extinction coefficients, two waveguides of the same material differing only in length may also show spectral absorbance features when comparing the two. To be certain that the features observed in absor-

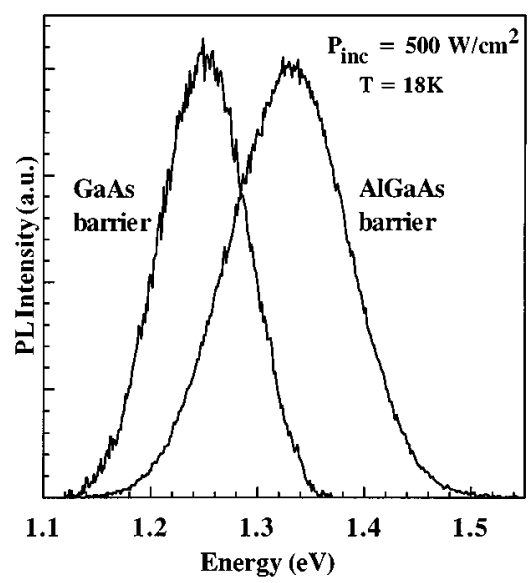

FIG. 3. Photoluminescence spectra of InAs/GaAs and InAs/Al ${ }_{0.15} \mathrm{Ga}_{0.85} \mathrm{As}$ quantum dots $\left(n=2 \times 10^{18} \mathrm{~cm}^{-3}\right)$.

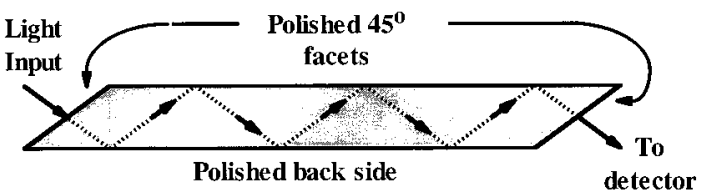

(a)

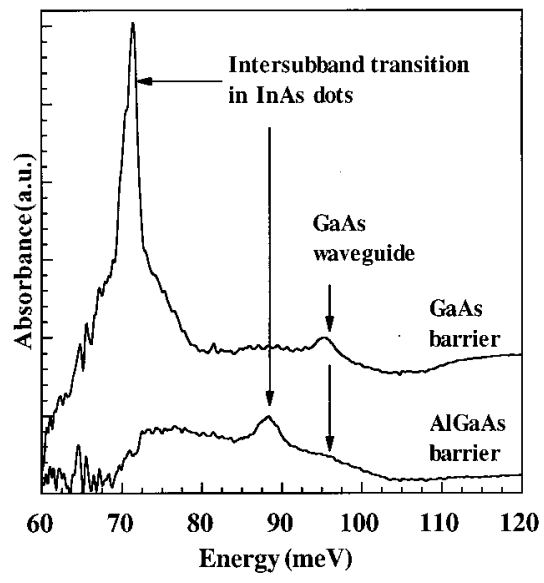

(b)

FIG. 4. Schematic of (a) multireflection waveguide geometry used for FTIR absorption measurements and (b) FTIR absorbance spectra of InAs quantum dots with $\mathrm{GaAs}$ and $\mathrm{Al}_{0.15} \mathrm{Ga}_{0.85}$ As barriers.

bance are not from the GaAs substrate, a comparison was made to the absorbance of the GaAs substrate with respect to air.

Measured absorbance spectra are shown in Fig. 4(b). For the sample grown with GaAs barriers, a strong peak is clearly observed at $70 \mathrm{meV}$ which we believe is due to intersubband absorption in the quantum dots. It should be noted that the cutoff of the measurement system (detector and beamsplitter) is at $50 \mathrm{meV}$ where the detected signal becomes weak. Because of this, the peak observed is not of true shape or absorbance value, though a strong enough signal is detected to be certain that the peak is truly an absorbance peak from the sample. For the sample grown with AlGaAs barriers, a peak at $89 \mathrm{meV}$ is observed which we believe to be due to intersubband absorption in the quantum dots. With the absence of distinguishable peaks in the PL spectra corresponding to excited state transitions in the quantum dots, it is not clear whether the far-infrared absorption is due to subband-subband or subband-continuum transitions. Additional peaks are observed which we will identify as follows. A sharp, but weak, peak is observed at $86 \mathrm{meV}$ corresponding to $\mathrm{CO}_{2}$ absorption from differing levels of the gases between measurements. A broad base-line peak is observed between $\sim 75$ and $100 \mathrm{meV}$, and a narrower peak at $97 \mathrm{meV}$ are due to spectral features occurring from waveguides of varying length, as confirmed by absorbance spectra of GaAs as compared to air.

In conclusion, we have examined the properties of directly Si-doped InAs/GaAs and InAs/ $/ \mathrm{Al}_{0.15} \mathrm{Ga}_{0.85} \mathrm{As}$ selforganized quantum dots. A blue shift in PL spectra peak intensity is observed with increasing doping for InAs/GaAs 
quantum dots, and a further blue shift for an increased barrier height in the case of InAs/ $\mathrm{Al}_{0.15} \mathrm{Ga}_{0.85} \mathrm{As}$ quantum dots. Farinfrared absorption peaks are observed at $70 \mathrm{meV}$ for InAs/ GaAs quantum dots and at $89 \mathrm{meV}$ for InAs/ $\mathrm{Al}_{0.15} \mathrm{Ga}_{0.85} \mathrm{As}$ quantum dots using a FTIR system and a multireflection waveguide geometry. The results of this work suggest that these dots provide a means of developing mid and farinfrared detectors which may be tuned with varying barrier heights.

This work is supported by the National Science Foundation under Grant No. ECS-9628973.

${ }^{1}$ B. F. Levine, III-V Quantum Wells and Superlattices, edited by P. Bhattacharya (INSPEC, London, 1996), p. 288.

${ }^{2}$ L. West and S. Eglash, Appl. Phys. Lett. 46, 1156 (1985).

${ }^{3}$ B. Levine, K. Choi, C. Bethea, J. Walker, and R. Malik, Appl. Phys. Lett. 50, 1092 (1987).

${ }^{4}$ Intersubband Transitions in Quantum Wells, edited by E. Rosencher, B. Vinter, and B. F. Levine (Plenum, New York, 1992).
${ }^{5}$ J. Faist, F. Capasso, D. Sivco, A. Hutchinson, C. Sirtori, and A. Y. Cho, Infrared Phys. Technol. 36, 99 (1995).

${ }^{6}$ C. Sirtori, J. Faist, F. Capasso, D. Sivco, A. Hutchinson, and A. Cho, Appl. Phys. Lett. 69, 2810 (1996).

${ }^{7}$ J. Singh, IEEE Photonics Technol. Lett. 8, 488 (1996).

${ }^{8}$ M. Grundmann, N. Ledentsov, R. Heitz, L. Eckey, J. Christen, J. Bohrer, D. Bimberg, S. Ruvimov, P. Werner, U. Richter, J. Heydenreich, V. Ustinov, A. Egorov, A. Zhukov, P. Kopev, and Z. Alferov, Phys. Status Solidi B 188, 249 (1995).

${ }^{9}$ K. H. Schmidt, G. Medeiros-Ribeiro, M. Oestreich, P. M. Petroff, and G. H. Dohler, Phys. Rev. B 54, 11346 (1996).

${ }^{10}$ K. Kamath, N. Chervela, K. K. Linder, T. Sosnowski, H.-T. Jiang, T. Norris, J. Singh, and P. Bhattacharya, Appl. Phys. Lett. 71, 927 (1997).

${ }^{11}$ K. Mukai, N. Ohtsuka, and M. Sugawara, Appl. Phys. Lett. 70, 2416 (1997).

${ }^{12}$ H. Drexler, D. Leonard, W. Hansen, J. P. Kotthaus, G. Medeiros-Ribeiro, and P. M. Petroff, Phys. Rev. Lett. 73, 2252 (1994).

${ }^{13}$ K. W. Berryman, S. A. Lyon, and M. Segev, Appl. Phys. Lett. 70, 1861 (1997).

${ }^{14}$ J. Lee, H. Lee, E. Shin, S. Yu, D. Kim, and G. Ihm, Appl. Phys. Lett. 70, 2885 (1997).

${ }^{15}$ X. Zhou, P. K. Bhattacharya, G. Hugo, S. C. Hong, and E. Gulari, Appl. Phys. Lett. 54, 855 (1989). 